\title{
lonic charging by local imbalance at interfaces in hybrid lead halide perovskites
}

\author{
Osbel Almora, Antonio Guerrero, and Germà Garcia-Belmonte ${ }^{\mathrm{a})}$ \\ Institute of Advanced Materials (INAM), Universitat Jaume I, 12071 Castelló, Spain
}

(Received 11 December 2015; accepted 19 January 2016; published online 28 January 2016)

\begin{abstract}
Identification of specific operating mechanisms becomes particularly challenging when mixed ionic-electronic conductors are used in optoelectronic devices. Ionic effects in perovskite solar cells are believed to distort operation curves and possess serious doubts about their long term stability. Current hysteresis and switchable photovoltaic characteristics have been connected to the kinetics of ion migration. However, the nature of the specific ionic mechanism (or mechanisms) able to explain the operation distortions is still poorly understood. It is observed here that the local rearrangement of ions at the electrode interfaces gives rise to commonly observed capacitive effects. Charging transients in response to step voltage stimuli using thick $\mathrm{CH}_{3} \mathrm{NH}_{3} \mathrm{PbI}_{3}$ samples show two main polarization processes and reveal the structure of the ionic double-layer at the interface with the non-reacting contacts. It is observed that ionic charging, with a typical response time of $10 \mathrm{~s}$, is a local effect confined in the vicinity of the electrode, which entails absence of net mobile ionic concentration (space-charge) in the material bulk. (C) 2016 AIP Publishing LLC.

[http://dx.doi.org/10.1063/1.4941033]
\end{abstract}

Interfaces play a central role in every kind of solar cell. Electronic issues about the energetics and kinetics at the interface should be considered for efficient charge extraction and carrier selectivity. For classical photovoltaic structures, modeling of contact assumes mobile electronic carriers and incorporates the influence of fixed ionic charges responsible for the doping character of semiconducting material layers. ${ }^{1}$ However, the understanding of other kinds of solar cells becomes more demanding when ionic charges are transported within light absorbing or electronic conducting layers, and finally alter the solar cell steady-state operation. There is one example of an optoelectronic device in which the interplay between electronic and ionic properties is determinant: in electrochemical light-emitting diodes, the ionic charge kinetics is essential to explain time evolution of the light power emission as functional materials are mixed ionicelectronic conductors. ${ }^{2}$ Also, in photovoltaics, perovskitebased solar cells constitute a special type of light-harvesting devices because, in addition to electronic mechanisms, the presence of moving ionic species is believed to modify current-voltage curves. ${ }^{3,4}$ Among other explanations related to either ferroelectricity ${ }^{5-7}$ or electronic trapping properties of perovskites, ${ }^{8}$ there is growing consensus that the ionic kinetics lies behind the commonly observed current-voltage hysteretic response, ${ }^{9-11}$ and switchable photovoltaic characteristics. ${ }^{12}$ Several studies have analyzed the defect chemistry of hybrid perovskite materials, and the inherent dependence of their doping character on the processing route. ${ }^{13,14}$ It is identified from first-principles computational analyses that iodine anions can easily migrate through a vacancy-assisted mechanism, although different calculated activation energies have been reported. ${ }^{10,11,15}$ Moreover, these studies observe the presence of several ionic vacancies

\footnotetext{
${ }^{\text {a) }}$ Author to whom correspondence should be addressed. Electronic mail: garciag@uji.es.Tel.: +34 964387538.
}

$\left(\mathrm{I}^{-}, \mathrm{Pb}^{2+}\right.$ and $\left.\mathrm{CH}_{3} \mathrm{NH}_{3}{ }^{+}\right)$in equilibrium at room temperature, being their final concentrations highly influenced by the particular synthesis procedure. However, the nature of the specific ionic mechanism (or mechanisms) able to explain the hysteretic behavior is still poorly understood. In order to advance into our comprehension of perovskite solar cell functioning, knowledge about ionic effects should rely on sound and informative experiments on interface operation. Here, we explore charging transients in response to step voltage stimuli using thick $\mathrm{CH}_{3} \mathrm{NH}_{3} \mathrm{PbI}_{3}\left(\mathrm{MAPbI}_{3}\right)$ samples, and observe two main polarization processes in the dark connected to the response of the double-layer interfacial structure: (i) Helmholtz layer formed at $\mathrm{MAPbI}_{3} /$ contact interfaces and (ii) electrode-induced local charge imbalance within the ion diffuse layer. It is demonstrated here that ionic charging is a local effect confined at the electrode vicinity. Local ionic rearrangement gives rise to measurable capacitances, which produce capacitive currents and concomitant hysteresis behavior as recently shown for hybrid lead iodide perovskite-based solar cells. ${ }^{16,17}$

With the aim of separating bulk from interface mechanisms, thick $\mathrm{MAPbI}_{3}$ pellets of different thickness (400$800 \mu \mathrm{m}$ ) are sandwiched between Au contacts (see Figure S1 in supplementary material). ${ }^{18}$ The synthesis of $\mathrm{CH}_{3} \mathrm{NH}_{3} \mathrm{PbI}_{3}$ was carried by slow evaporation in gamma-butyrolactone containing stoichiometric amounts of methyl ammonium iodide and lead iodide. The mixture was placed in an open crystallization dish into a well-ventilated oven at $170^{\circ} \mathrm{C}$ during $3 \mathrm{~h}$. The black powder was characterized by XRD to confirm the pure perovskite crystallographic structure (Figure S2). ${ }^{18}$ SEM analysis also shows a highly crystalline nature of the powder [Figure 1(a)]. The disks were prepared by pressing at 6 ton $\mathrm{CH}_{3} \mathrm{NH}_{3} \mathrm{PbI}_{3}$ powder into a $1.33 \mathrm{~cm}$ diameter cylinder pellet die without any subsequent temperature annealing. The mass and the respective thickness of the prepared pellets are listed in Table S1. ${ }^{18}$ Samples, which form a compact, crack-free 

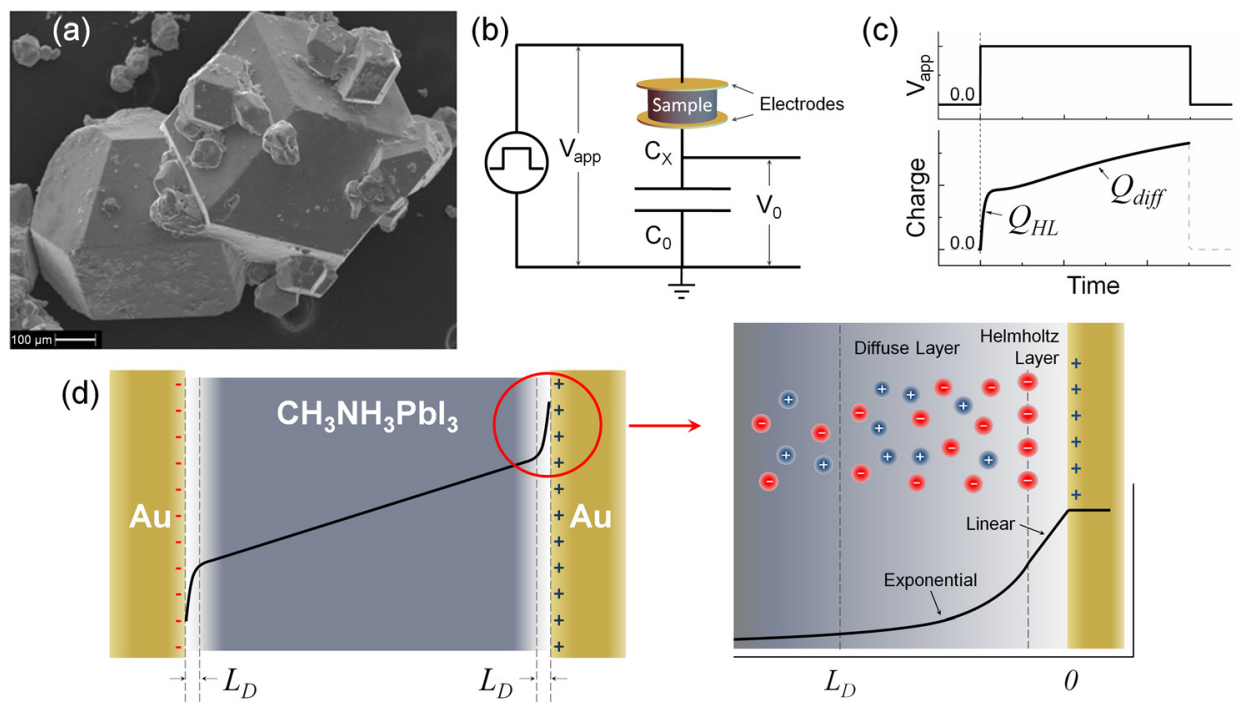

FIG. 1. (a) SEM image of large $\mathrm{CH}_{3} \mathrm{NH}_{3} \mathrm{PbI}_{3}$ crystals forming the studied samples. (b) Sawyer-Tower circuit for measurements. (c) Applied rectangular potential pulse of height $V_{\text {app }}$ (Agilent 33220A $2 \mathrm{MHz}$ function arbitrary waveform generator) in the ST set-up and general shape of the signal $V_{0}\left(Q=C_{0} V_{0}\right)$ obtained with $\mathrm{MAPbI}_{3}$ pellets, registered with Hewlett Packard Infinium oscilloscope $(500 \mathrm{MHz}, 1 \mathrm{GSa} / \mathrm{s})$. Two different transients (time not in scale) with their respective steps are observed that correspond to the exponential terms in Equation (1). (d) Schematic representation of the voltage distribution within the sample (not in scale), and detail of the double-layer interfacial structure. The Helmholtz compact layer behaves as a planar capacitor (linear potential drop), while the diffuse layer exhibits an exponential voltage drop.

pellet, are made up of large single crystals with the aim of reducing the concentration of ionic defects formed at grain boundaries [Figure S3]. ${ }^{18}$ Samples were directly transferred to a Quatro Cryosystem vacuum chamber $\left(2.0 \times 10^{-3} \mathrm{mbar}\right)$ from Novocontrol in order to evacuate surface absorbed water. Our aim here is discerning whether ionic transport through long distances is responsible for the measured kinetics of charging in the $10 \mathrm{~s}$ time scale, and the extent of the ionic space charge. Hence, charging time can be used as a discriminating test among different polarization mechanisms.

Charging transients are registered by means of the Sawyer-Tower circuit (ST), ${ }^{19,20}$ which is the classical set-up typically used to explore hysteretic loops of ferroelectric materials. In the configuration of the circuit in Figure 1(b), a voltage signal $V_{\text {app }}$ is applied to the sample $C_{x}$ across a reference linear capacitor $C_{0}\left(C_{x} \ll C_{0}\right)$ connected in series, thereupon the charge in $C_{x}$ (as in $C_{0}$ ) is $Q=C_{0} V_{0}, V_{0}$ being the potential drop across the reference capacitor that is monitored with a high input impedance oscilloscope. Related experiments on $\mathrm{CH}_{3} \mathrm{NH}_{3} \mathrm{PbI}_{3-x} \mathrm{Cl}_{x}$ based cells using ST circuit have shown in previous works ${ }^{16,21}$ the absence of ferroelectric behavior at room temperature. Alternatively to the ST circuit, dielectric responses are better checked by using other techniques as impedance spectroscopy for higher accuracy in the high-frequency (short time) range. We investigate charging of a set of $\mathrm{MAPbI}_{3}$ pellets (Table S1) ${ }^{18}$ in response to step polarization voltages. By applying rectangular pulses of height $V_{\text {app }}$ [Figure 1(c)] into the ST set-up, the signal $V_{0}(t)$ in the reference capacitor presents a general shape, which is the combination of two exponentials with different characteristic times [Figure 1(c)]. Such a general form of the induced charge can be fitted to the following double exponential equation:

$$
Q(t)=Q_{\mathrm{HL}}\left[1-\exp \left(-t / \tau_{\mathrm{HL}}\right)\right]+Q_{\text {diff }}\left[1-\exp \left(-t / \tau_{\text {diff }}\right)\right],
$$

Each exponential term in Equation (1) can be associated with its respective step in the general shape of the signal. As discussed next, the first exponential term is associated with the Helmholtz layer dielectric features (represented by the parameters $Q_{\mathrm{HL}}$ and $\tau_{\mathrm{HL}}$ ), while the second step results from ionic charging at the diffuse layer contacting the electrode (with parameters $Q_{\text {diff }}$ and $\tau_{\text {diff }}$ ). These two polarization mechanisms constitute the double-layer structure [Figure 1(d)] electrical response and rely upon several recent proposals about the role of mobile ions as defects, ${ }^{9,22}$ in the operation of $\mathrm{MAPbI}_{3}$ based solar cells. Particularly, iodide anions have been identified to contribute as derived from first-principles simulation analyses. ${ }^{10,11,15}$ On the other hand, we carefully checked if the reference capacitor has an influence on the general response in Figure 1(c). We verified that the $C_{0}$ values introduce only minor distortions in the form of slight undershoots with characteristic time $\tau_{\mathrm{HL}}<\tau_{\text {ref }}<\tau_{\text {diff }}$ (see Figure S4). ${ }^{18}$ Nevertheless, double-layer charge contributions are not affected by set-up induced small distortions.

It is first explored the faster rise of the charge signal through appropriate narrow time windows in Figure 2(a). After fitting using Equation (1), charges of order $\mathrm{nC} \mathrm{cm}^{-2}$ are induced [Figure 2(c)]. As explained below, this amount of charge can be hardly caused by dielectric polarization of the bulk $\mathrm{MAPbI}_{3}$ material. Dielectric geometrical capacitances $C_{g} \sim 27 \mathrm{pF} \mathrm{cm}^{-2}$ are expected for an $800 \mu$ m-thick pellet (confirmed using impedance spectroscopy in Figure S5, ${ }^{18}$ in the high frequency part of the spectra), which entails polarization charges approximately equal to $0.1 \mathrm{nC} \mathrm{cm}^{-2}$ for $V_{\text {app }}=5 \mathrm{~V}$ using $Q=C_{g} V_{\text {app }}$. The previous discrepancy implies that the fast charging component cannot be originated by dielectric bulk properties of the $\mathrm{MAPbI}_{3}$ material. Instead, fast charging signal can be interpreted in terms of the capacitance of the Helmholtz layer dielectric features, as depicted in Figure 1(d) with $C_{\mathrm{HL}}=\varepsilon \varepsilon_{0} / d$, where $\varepsilon$ is the dielectric constant of the material, $\varepsilon_{0}$ stands for the vacuum permittivity, 


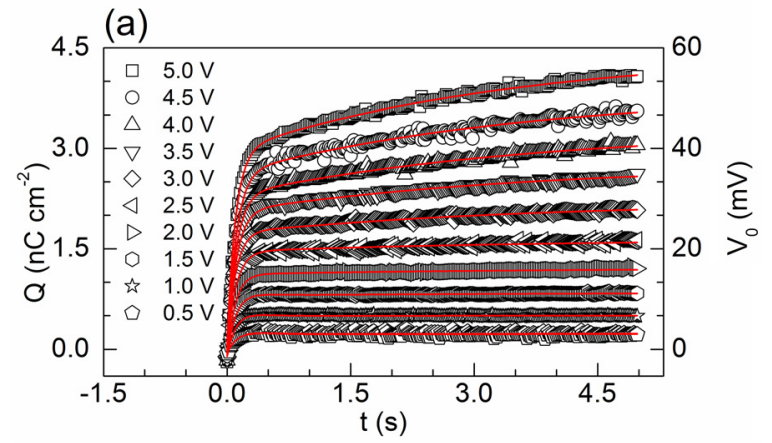

(b)

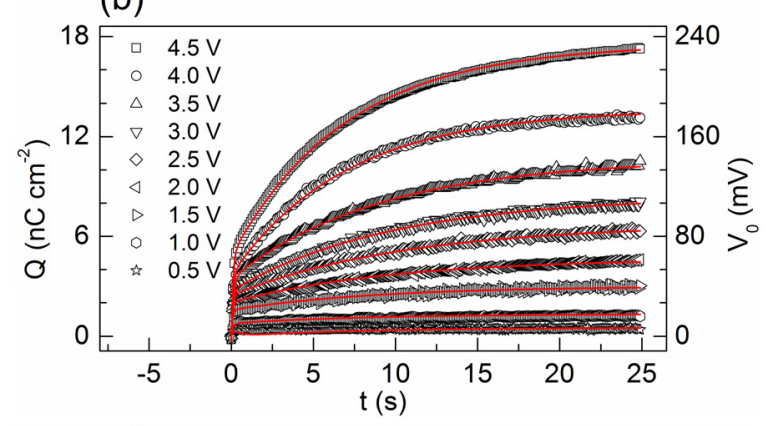

(c)

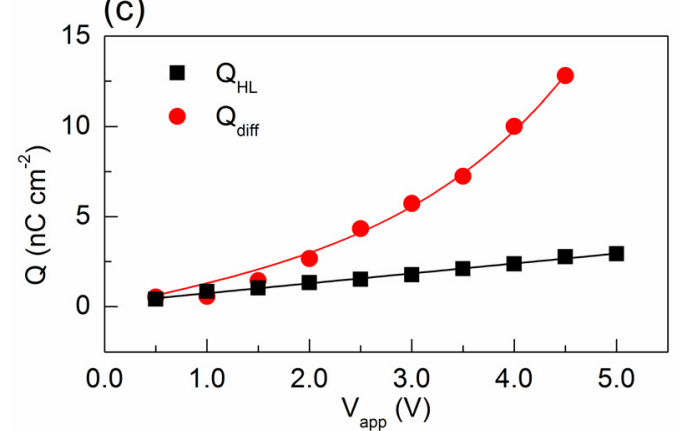

FIG. 2. Example of $Q$ (and $V_{0}$ ) signals of an $800 \mu \mathrm{m}$-thick $\mathrm{MAPbI}_{3}$ pellet $\left(1.33 \mathrm{~cm}^{2}\right.$ of active area) for different $V_{\text {app }}$ with $C_{0}=100 \mathrm{nF}$ for short (a) and long (b) measuring times. Points correspond to experimental data and the solid red lines the fitting with Equation (1). (c) Values of $Q_{\mathrm{HL}}$ with respect to $V_{\text {app }}$ extracted from fitting using Equation (1), showing a linear trend (solid line) in accordance with a plane capacitor behavior. Ionic charge $Q_{\text {diff }}$ values, extracted from fitting using Equation (1), following fitting of Equation (2) (solid line).

and $d$ represents the effective separation of the Helmholtz layer. High capacitances are expected here of the order of $1-10 \mu \mathrm{F} \mathrm{cm}{ }^{-2}$ in accordance with low-frequency capacitance values (Figure S5). ${ }^{18}$ Different thicknesses and areas are checked for consistency (Figure S6). ${ }^{18}$

Longer decay times arise in a more evident way opening the measuring window in Figure 2(b). Slow dynamic processes on the order of ten seconds have been identified earlier ${ }^{4,22-25}$ in transient current and photovoltage decays, and related to the $\mathrm{MAPbI}_{3}$ structural and interface properties, even for much thinner $(<1 \mu \mathrm{m})$ samples. Here, we associate the slower exponential charge increase with the defect ionic rearrangement at the diffuse layer contacting electrode interfaces [Figure 1(d)]. ${ }^{16}$ The induced charges in Figure 2(c) attain larger values than those resulting from the fast component but clearly deviate from a linear trend. Gouy-Chapman theory $^{26}$ for the diffuse layer structure [Figure 1(d)] assumes Poisson equation and Boltzmann distribution in the meanfield approach resulting in a charge per unit area as

$$
Q_{\mathrm{diff}}=\frac{2 \varepsilon \varepsilon_{0} k_{\mathrm{B}} T}{q L_{D}} \sinh \left(\frac{q \Delta \varphi}{2 k_{\mathrm{B}} T}\right),
$$

where $k_{\mathrm{B}}$ is the Boltzmann constant, $T$ the absolute temperature, $q$ the elementary charge, $\Delta \varphi$ is the potential drop between the metallic contact and the electrolyte bulk, and $L_{D}$ accounts for the ionic Debye length

$$
L_{D}=\sqrt{\frac{\varepsilon \varepsilon_{0} k_{\mathrm{B}} T}{q^{2} N}} .
$$

Here, $N$ is the total density of mobile ions (both positive and negative defects) forming the interfacial space charge of the diffuse layer. The analysis is however unable to distinguish among different ionic defects. By derivation, Equation (2) reduces to the Debye differential capacitance $C_{D}$ when $\Delta \varphi=0$. It should be recognized that Eq. (2) was originally devised for liquid electrolytes while the reported experiments involve ionic defects as transport species in a solid electrolyte. Our proposal is considered then as a starting point to progress in the otherwise complex problem of ionic electrode polarization in the solid state.

The fitting of Equation (2) to $Q_{\text {diff }}$ values [solid line in Figure 2(c)] shows good agreement with the model. Because only a portion of $V_{\text {app }}$ drops at the diffuse layer, the model in Equation (2) assumes a rescaling factor $m\left(\Delta \varphi=V_{\text {app }} / m\right)$ as a free, voltage-independent fitting parameter. The resulting value for a single interface is $L_{D}=236 \pm 15 \mathrm{~nm}$. It is also inferred that for the whole sample $m=36 \pm 2$, which means that approximately $3 \%$ of $V_{\text {app }}$ drops in a region in the vicinity of the electrodes $(0.06 \%$ of the sample thickness), as illustrated in Figure 1(d). This potential distribution allows explaining why the bulk properties dominate over electrode polarization features in impedance response of thick samples (Figure S8) at high frequencies. ${ }^{18}$ The ionic charge concentration calculated by mean of Equation (3) results in $N=3.9 \times 10^{13} \mathrm{~cm}^{-3}$. As mentioned above, this is the total density, which includes all types of ionic defects, either positively or negatively charged. A relatively low defect concentration is found in the samples, which is in agreement with our preparation method that allows synthesizing large $\mathrm{MAPbI}_{3}$ crystals [Figure 1(a)] and largely reduces grain boundary defects. Thin $\mathrm{MAPbI}_{3}$ samples with smaller crystal sizes will produce greater ionic defect density $N \sim 10^{17} \mathrm{~cm}^{-3}$ with reduced $L_{D} \sim 10 \mathrm{~nm}$ as recently reported. ${ }^{16}$ This allows comparing our findings using thick samples with those values obtained for perovskite solar cells with film thickness of several hundred nanometers. In both cases, the ionic space charge zone is confined in a relatively narrow layer contacting the electrode, i.e., $L_{D} / L \ll 1$. We note here that double layer interface charging may be altered in those cases in which a strong chemical interaction occur between $\mathrm{MAPbI}_{3}$ and the contacting materials. Instead of Debye capacitances, the interface polarization is dominated by the complex interaction between electronic and ionic species at both sides of the contact. ${ }^{27,28}$ Our experiments can then be considered as examples of the response of non-reacting contacts.

Finally, the kinetics of slow ionic charging at the diffuse layer of characteristic response time of order $\tau_{\text {diff }}=10 \mathrm{~s} \mathrm{can}$ 
be used to estimate the ion diffusivity value. The problem of ion charging dynamics constitutes a well-established issue in the field of liquid electrolytes and colloids. ${ }^{29}$ It is usually distinguished between two time scales: $\tau_{D}=L_{D}^{2} / D$ and $\tau_{L}=L^{2} / D, D$ being the ion diffusion coefficient. These two time scales, related to either the Debye screening length $L_{D}$ or the sample thickness $L$, rarely correlate to the observed response time. Instead, the diffuse layer charging appears to be determined by their harmonic mean $\tau_{\text {diff }}=\sqrt{\tau_{D} \tau_{L}}$. Therefore, the ionic diffusion coefficient can be readily estimated from the charging time $\tau_{\text {diff }}$ by means of the following expression: ${ }^{29}$

$$
\tau_{\text {diff }}=\frac{L_{\mathrm{D}} L}{D} .
$$

This relationship captures the intuitive idea about the necessary bulk ion transport to build up the diffuse layer, while keeping the ion rearrangement as a mechanism thermodynamically determined at $L_{D}$ short ranges. This view agrees with the equivalent circuit $R C$ model that comprises a bulk resistance in series with the electrode polarization capacitance, and with the first-order exponential responses of Fig. 2. It is also recognized that other response-time dependences may appear particularly in the presence of Faradaic currents (non-blocking or reacting electrodes) or high applied voltages when large extent space-charge regions enter the electrolyte bulk. ${ }^{29}$ Even charging oscillations have been observed as with ionic liquids, ${ }^{30-32}$ although our experiments exhibit a simpler exponential shape. In any case, Equation (4) allows extracting the order of magnitude of an ionic transport parameter. One can obtain $D \approx 4 \times 10^{-7} \mathrm{~cm}^{2} \mathrm{~s}^{-1}$, a value much higher than ionic diffusivities calculated from indirect methods, ${ }^{11,33}$ but of similar order than those extracted from much longer voltage transients. ${ }^{9}$ We note here that charging kinetics of order $10 \mathrm{~s}$ cannot be explained solely in terms of ion diffusion across the material bulk. Long distance ionic transport ( $\sim 400 \mu \mathrm{m}$ assuming half of the bulk diffusion path) would entail unrealistic ionic diffusivities $\left(D=L^{2} / \tau_{\text {diff }} \approx 10^{-4} \mathrm{~cm}^{2} \mathrm{~s}^{-1}\right)$. If ions are accumulated by drift through the material bulk, mobility values should be as high as $\mu=L^{2} / \tau_{\text {diff }} V_{\text {app }} \approx 3 \times 10^{-5} \mathrm{~cm}^{2} \mathrm{~V}^{-1} \mathrm{~s}^{-1}$ (for $V_{\text {app }}=5 \mathrm{~V}$ ), again unphysical values for ionic species. These last comments reinforce our claim about the origin of the electrode polarization mechanism. Space charge occurs by a local imbalance of characteristic size equaling the ionic Debye length, with a kinetics coupled with the ion bulk transport. Charging then is restricted in the vicinity of the electrode under the regime $L_{D} / L \ll 1$, which predicts nonexistent ionic gradient concentration in the material bulk.

In summary, the ST circuit allows checking the charging response of thick $\mathrm{MAPbI}_{3}$ samples. This procedure has revealed the electrical structure of the interfacial doublelayer formed by mobile ions at non-interacting Au contacts. Two different charging mechanisms have been identified linked with the Helmholtz and diffuse layers. It is discussed how the charging is governed by the local imbalance at the interface with an extension equaling the ionic Debye length that depends on the ionic defect concentration. Our approach highlights the mixed-conductor character of lead halide perovskites and establishes solid basis for solar cell modeling and experiment interpretation.

We thank financial support from MINECO of Spain under Project (MAT2013-47192-C3-1-R), and Generalitat Valenciana (Prometeo/2014/020). O.A. acknowledges Generalitat Valenciana for a Grant (GRISOLIAP2014/035). SCIC at UJI are also acknowledged.

${ }^{1}$ S. J. Fonash, Solar Cell Device Physics, 2nd ed. (Elsevier, Burlington, 2010).

${ }^{2}$ M. Lenes, G. Garcia-Belmonte, D. Tordera, A. Pertegás, J. Bisquert, and H. J. Bolink, Adv. Funct. Mater. 21, 1581 (2011).

${ }^{3}$ R. Gottesman, E. Haltzi, L. Gouda, S. Tirosh, Y. Bouhadana, A. Zaban, E. Mosconi, and F. De Angelis, J. Phys. Chem. Lett. 5, 2662-2669 (2014).

${ }^{4}$ W. Tress, N. Marinova, T. Moehl, S. M. Zakeeruddin, M. K. Nazeeruddin, and M. Grätzel, Energy Environ. Sci 8, 995 (2015).

${ }^{5}$ J. M. Frost, K. T. Butler, and A. Walsh, APL Mater. 2, 081506 (2014).

${ }^{6}$ J. Wei, Y. Zhao, H. Li, G. Li, J. Pan, D. Xu, Q. Zhao, and D. Yu, J. Phys. Chem. Lett. 5, 3937-3945 (2014).

${ }^{7}$ H.-W. Chen, N. Sakai, M. Ikegami, and T. Miyasaka, J. Phys. Chem. Lett. 6, 164-169 (2015).

${ }^{8}$ Y. Shao, Z. Xiao, C. Bi, Y. Yuan, and J. Huang, Nat. Commun. 5, 5784 (2014).

${ }^{9}$ T.-Y. Yang, G. Gregori, N. Pellet, M. Grätzel, and J. Maier, Angew. Chem. 127, 8016 (2015).

${ }^{10}$ J. M. Azpiroz, E. Mosconi, J. Bisquert, and F. De Angelis, Energy Environ. Sci. 8, 2118 (2015).

${ }^{11}$ C. Eames, J. M. Frost, P. R. F. Barnes, B. C. O'Regan, A. Walsh, and M. Saiful Islam, Nat. Commun. 6, 7497 (2015).

${ }^{12}$ Z. Xiao, Y. Yuan, Y. Shao, Q. Wang, Q. Dong, C. Bi, P. Sharma, A. Gruverman, and J. Huang, Nat. Mater. 14, 193 (2015).

${ }^{13}$ W.-J. Yin, T. Shi, and Y. Yan, Appl. Phys. Lett. 104, 063903 (2014).

${ }^{14}$ J. Kim, S.-H. Lee, J. H. Lee, and K.-H. Hong, J. Phys. Chem. Lett. 5, 1312-1317 (2014).

${ }^{15}$ J. Haruyama, K. Sodeyama, L. Han, and Y. Tateyama, J. Am. Chem. Soc. 137, 10048-10051 (2015).

${ }^{16}$ O. Almora, I. Zarazua, E. Mas-Marza, I. Mora-Sero, J. Bisquert, and G. Garcia-Belmonte, J. Phys. Chem. Lett. 6, 1645-1652 (2015).

${ }^{17}$ B. Chen, M. Yang, X. Zheng, C. Wu, W. Li, Y. Yan, J. Bisquert, G. GarciaBelmonte, K. Zhu, and S. Priya, J. Phys. Chem. Lett. 6, 4693-4700 (2015).

${ }^{18}$ See supplementary material at http://dx.doi.org/10.1063/1.4941033 for more experimental details, results, and discussions.

${ }^{19}$ C. Sawyer and C. Tower, Phys. Rev. 35(3), 269 (1930).

${ }^{20}$ K. M. Rabe, C. H. Ahn, and J.-M. Triscone, in Topics in Applied Physics, edited by C. E. Ascheron (Springer Verlag, Berlin/Heidelberg, 2007), p. 388.

${ }^{21}$ M. Coll, A. Gomez, E. Mas-Marza, O. Almora, G. Garcia-Belmonte, M. Campoy-Quiles, and J. Bisquert, J. Phys. Chem. Lett. 6(8), 1408 (2015).

${ }^{22}$ W. H. Nguyen, C. D. Bailie, E. L. Unger, and M. D. McGehee, J. Am. Chem. Soc. 136(31), 10996 (2014).

${ }^{23}$ R. S. Sanchez, V. Gonzalez-Pedro, J.-W. Lee, N.-G. Park, Y. S. Kang, I. Mora-Sero, and J. Bisquert, J. Phys. Chem. Lett. 5(13), 2357 (2014).

${ }^{24}$ A. Baumann, K. Tvingstedt, M. C. Heiber, S. Väth, C. Momblona, H. J. Bolink, and V. Dyakonov, APL Mater. 2(8), 081501 (2014).

${ }^{25}$ J. Beilsten-Edmands, G. E. Eperon, R. D. Johnson, H. J. Snaith, and P. G. Radaelli, Appl. Phys. Lett. 106(17), 173502 (2015).

${ }^{26}$ V. S. Bagotsky, Fundamentals of Electrochemistry (Wiley, Hoboken, 2005), pp. 705.

${ }^{27}$ C. R. Mariappan, T. P. Heins, and B. Roling, Solid State Ionics 181, 859 (2010).

${ }^{28}$ Y. Kato, L. K. Ono, M. V. Lee, S. Wang, S. R. Raga, and Y. Qi, Adv. Mater. Interfaces 2, 1500195 (2015).

${ }^{29}$ M. Z. Bazant, K. Thornton, and A. Ajdari, Phys. Rev. E 70, 021506 (2004).

${ }^{30}$ M. Z. Bazant, B. D. Storey, and A. A. Kornyshev, Phys. Rev. Lett. 106, 046102 (2011).

${ }^{31}$ A. A. Lee, S. Kondrat, D. Vella, and A. Goriely, Phys. Rev. Lett. 115, 106101 (2015).

${ }^{32}$ X. Jiang, J. Huang, H. Zhao, B. G. Sumpter, and R. Qiao, J. Phys.: Condens. Matter 26, 284109 (2014).

${ }^{33}$ M. Bag, L. A. Renna, R. Y. Adhikari, S. Karak, F. Liu, P. M. Lahti, T. P. Russell, M. T. Tuominen, and D. Venkataraman, J. Am. Chem. Soc. 137, 13130 (2015). 